\title{
Backstepping Controller Design for a Kind of High Speed Vehicle
}

\author{
Wenguang Zhang, Zijian Lin and Junwei Lei
}

Department of control engineering, Naval Aeronautical and Astronautical University, Yantai, 264001, China

leijunwei@126.com

Keyword: Backstepping, Stability, Lyapunov function, Adaptive, Robustness

\begin{abstract}
A kind of backstepping controller is designed for a kind of pitch channel model of hypersonic aircrafts. The main task is to realize attack angle tracking of hypersonic aircrafts. Compared with traditional linear design method, the main difference is that the nonlinear model is directly used in the design process by adopting a Lyapunov function method to guarantee the stability of the whole system. At last, detailed simulations are done to show the rightness of the proposed method.
\end{abstract}

\section{Introduction}

Today hypersonic weapon technology is an important development direction about which the major military powers are concerned [1].High Mach number aircraft is not only effective in high-altitude, high-speed penetration and can combat the enemy directly, but also can be used as a platform for long-range raid weapons, which improves the effectiveness of long-range combat. Inversion of control design method, due to its unique construction and design process for unmatched uncertainties of processing power, has been successfully applied in aircrafts, missiles, motors, robots. On the basis of hypersonic vehicle PID control, this paper carries out the inversion controller design, and changes its control law parameters to make the control model to achieve the effect of PID control and at the same time the simulation curve can quickly and smoothly to achieve the desired value quickly and smoothly.

\section{Model Description}

Considering the elastic shape structure, a kind of pitch channel hypersonic aircraft model built according to Lagrange equation is released by USA air force as followed:

$$
\begin{gathered}
\dot{V}=\frac{T \cos \alpha-D}{m}-g \sin \gamma \\
\dot{\phi}=-2 \varsigma \omega_{n} \phi-\omega_{n}^{2} \phi+\omega_{n}^{2} \phi_{c} \\
\dot{\gamma}=\frac{L+T \sin \alpha}{m V}-\frac{g \cos \gamma}{V} \\
\dot{\alpha}=q-\dot{\gamma} \\
\dot{q}=\frac{M}{I} \\
\dot{h}=V \sin \gamma \\
\ddot{\eta}_{i}=-2 \varepsilon_{m} \omega_{m i} \dot{\eta}_{i}-\omega_{m i}^{2} \eta_{i}+N_{i}
\end{gathered}
$$

Where

$$
T=\bar{q} s\left(C_{T \phi} \phi+C_{T}+C_{T}^{\eta} \eta\right) \quad, \quad D=\bar{q} S C_{D}
$$

And $V$ is speed, $\gamma$ is the speed angle, $\alpha$ is attack angle, $Q$ is the attitude angle speed, $h$ is the height, $\phi$ is the oil supplying factor, $\delta_{c}$ is the duck wing and $\delta_{e}$ is the lift rudder. 


\section{Back-stepping Controller Design}

An aircraft model formula can be obtained:

$$
\begin{gathered}
\dot{\alpha}=q-\dot{\gamma} \\
\dot{e}_{\alpha}=q-\dot{\gamma} \\
\dot{q}=\frac{M}{I}=\frac{M}{I_{y y}}=\frac{q_{b} s c_{b}\left(c_{m \alpha}+c_{m \delta}+c_{m q}\right)}{-8.03 * 10^{-4} m^{2}+219.74 m-1690000}
\end{gathered}
$$

Suppose $\dot{q}=k \delta+\delta_{a}$, in which $\delta_{a}$ is the intermediate variables.

$$
\begin{gathered}
e_{\alpha}=\alpha-\alpha^{d} \\
\dot{e}_{\alpha}=q-\dot{\gamma}=-\dot{\gamma}+q
\end{gathered}
$$

Make $q \rightarrow q^{d}$.

Suppose

$$
q^{d}=\dot{\gamma}-5 e_{\alpha}-0.2 \int e_{\alpha} d t-5 \dot{\alpha}
$$

Deduced

$$
\dot{q}^{d}=\ddot{\gamma}-5 \dot{\alpha}-0.2 e_{\alpha}
$$

Because

$$
\begin{gathered}
q-q^{d}=e_{q} \\
\dot{e}_{q}=\dot{q}-\dot{q}^{d}=\frac{M}{I}-\dot{q}^{d}=\frac{\bar{q} S \bar{c}\left[C_{M \alpha}+C_{M \delta}+C_{M q}\right]}{I_{y y}}-\dot{q}^{d} \\
=\frac{\bar{q} S \bar{c}\left[C_{M \alpha}+C_{M \delta}+0.0292(\delta-\alpha)\right]}{I_{y y}}-\dot{q}^{d} \\
=\frac{\bar{q} S \bar{c}\left[C_{M \alpha}+C_{M \delta}\right]+\bar{q} S \bar{c}[0.0292(\delta-\alpha)]}{I_{y y}}-\dot{q}^{d} \\
=\frac{\bar{q} S \bar{c}\left[C_{M \alpha}+C_{M \delta}\right]+0.0292 \bar{q} S \bar{c} \delta-0.0292 \bar{q} S \bar{c} \alpha}{I_{y y}}-\dot{q}^{d} \\
=\frac{0.0292 \bar{q} S \bar{c}}{I_{y y}} \delta+\frac{\bar{q} S \bar{c}\left[C_{M \alpha}+C_{M \delta}\right]-0.0292 \bar{q} S \bar{c} \alpha}{I_{y y}}-\dot{q}^{d}
\end{gathered}
$$

Because

$$
\dot{e}_{q}=\frac{0.0292 \bar{q} S \bar{c}}{I_{y y}} \delta+\frac{\bar{q} S \bar{c}\left[C_{M \alpha}+C_{M \delta}\right]-0.0292 \bar{q} S \bar{c} \alpha}{I_{y y}}-\dot{q}^{d}
$$

Hope $\delta$ design makes equal to $\delta_{a}$.

Suppose

$$
\delta_{a}=-40 e_{q}-0.02 \int e_{q} d t
$$

So we design

$$
\frac{0.0292 \bar{q} S \bar{c}}{I_{y y}} \delta=-\frac{\bar{q} S \bar{c}\left[C_{M \alpha}+C_{M \delta}\right]-0.0292 \bar{q} S \bar{c} \alpha}{I_{y y}}+\dot{q}^{d}+\delta_{a}
$$

Make $k=\frac{0.0292 \bar{q} S \bar{c}}{I_{y y}}$, suppose 


$$
y=\frac{\bar{q} S \bar{c}\left[C_{M \alpha}+C_{M \delta}\right]-0.0292 \bar{q} S \bar{c} \alpha}{I_{y y}}
$$

Then

$$
k \delta=-y+\dot{q}^{d}+\delta_{a}
$$

The control law is:

$$
\delta=\frac{-y+\dot{q}^{d}+\delta_{a}}{k}
$$

\section{Simulation Results}

Write the program with the conclusion derived from the formula. Change the control structure of the original program, so that the model can achieve the ideal value. So, I am continuously adjusting the assumption values of $\delta_{a}$ and $q^{d}$ in the process. While adjusting the values of $\delta_{a}$, I use the inversion control method and constantly change the parameters, so that the model can achieve its desired value.

According to the formula derived from the upper section of the control rate I rewrite the model for hypersonic vehicle.

The final flight simulation curve is as follows:

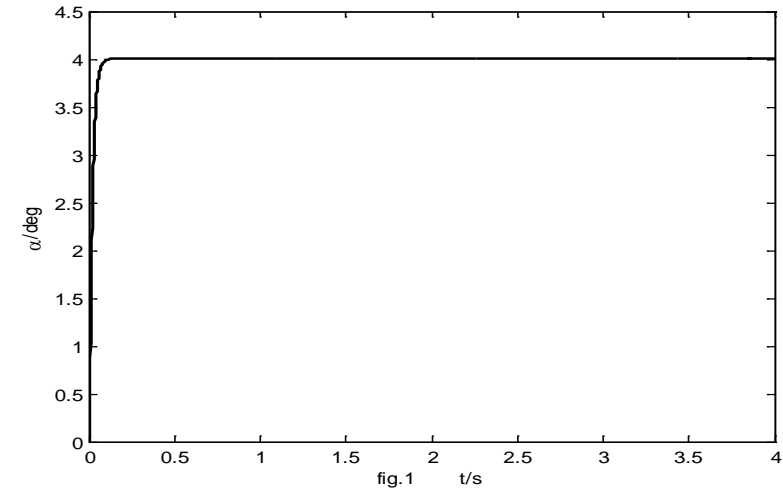

Fig. 1 Angle of attack curve

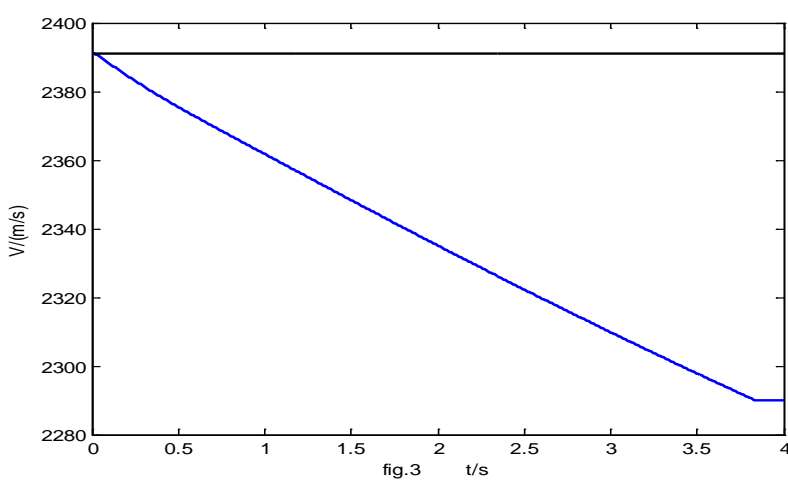

Fig. 3 Velocity curve

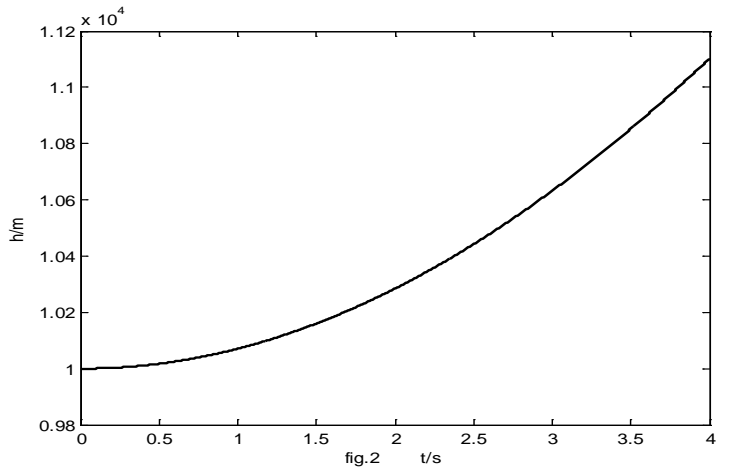

Fig. 2 Height curve

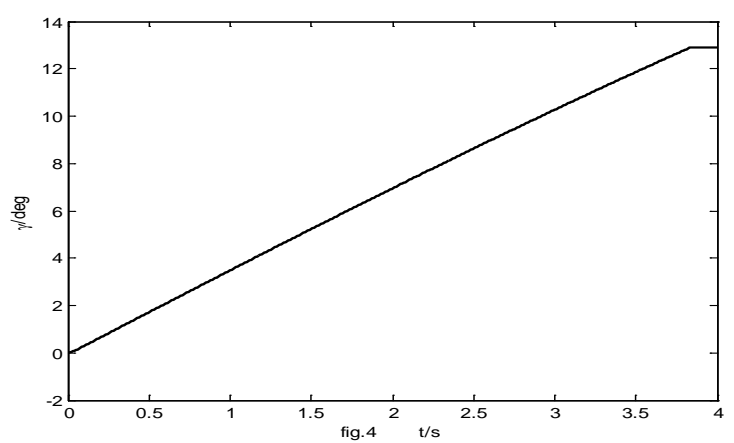

Fig. 4 Speed inclination curve

From above picture, it can be seen that in the simulation model, changing its control method and changing the control amount can accelerate the reaction time of the missile, and therefore the missile can quickly achieve the desired value and be stabilized. This indicates that using the inversion control can ensure the stability of the flight control system. This method is theoretically feasible. 


\section{Conclusions}

Based on the nonlinear model of hypersonic aircraft pitch channel, this paper gives its angle of attack tracking controller for a class of inverse design method of control, and verifies the correctness and validity of the method by using digital stimulation. But we did not study further about the interference immunity of the inversion vehicle under the disturbances of model perturbation, uncertainty, wind and moment. We will conduct further studies in the future.

\section{References}

[1]Appleby B.D and Adams N.J. Robust Estimator Design Applied to the Lateral Dynamics of a Hypersonic Vehicle, Proceedings of the AIAA Guidance, Navigation, and Control Conference and Exhibit. AIAA 1991-2689

[2]Vu P and Biezad D.J, Direct-lift Design Strategy for Longitudinal Control of Hypersonic Aircraft, Journal of guidance, control and dynamics,1994,1 7(6):1260-1266

[3]Marrison C.I and Stengel R.F, Synthesis of Robust Control Systems for a Hypersonic Aircraft, Proceedings of the 33rd IEEE Conference on Conference and Decision and Control,1994,3324-3329

[4]M. Ohno, Y. Yamaguchi, T. Hata, M. Takahama, Robust flight control law design for an automatic landing flight experiment [J], Control Engineering Practice, 1999, 7(9):1143-1152

[5]Naidu S D, Banda S. S. and Buffington J. L. Unified approach to $\mathrm{H}_{2}$ and $\mathrm{H}_{\infty}$ optimal control of a hypersonic vehicle [A].In: Proceedings of the American control conference[C].San Diego, California, 1999, vol.4:2737-2741.

[6]Ohno M, Yamaguchi Y, Hata T, et al. Robust flight control law design for an automatic landing flight experiment [J]. Control Engineering Practice, 1999, 7 (9):1143-1152.

[7]Heller M, Holzapfel F and Sachs G, Robust Lateral Control of Hypersonic Vehicles, Proceedings of the AIAA Guidance, Navigation, and Control Conference and Exhibit, Denver, CO, AIAA 2000-4248

[8]Heller M. and Sachs G., Flight dynamics and robust control of a hypersonic test vehicle with ramjet propulsion[A]. In: AIAA International Space Planes and Hypersonic Systems and Technologies Conference[C]. Norfolk, VA, AIAA 98-1521

[9]Johnson E. N., Limited Authority Adaptive Flight Control [D], Atlanta, Georgia Institute of Technology, 2000

[10]Johnson EN, Calise AJ, El-Shirbiny HA, and et al, Feedback Linearization with Neural Network Augmentation applied to X-33 Attitude Control [A] In: AIAA

[11]Guidance, Navigation and Control Conference[C], Denver: AIAA, 2000: 1-11

[12]Johnson EN, and Calise AJ, Limited Authority Adaptive Flight Control for Reusable Launch Vehicles [J], AIAA Journal of Guidance, Control, and Dynamics, 2003, 26(6):906-913 